mainly nonseminomatous tumors. The link between testicular cancer and gynecomastia may be often missed, resulting in delayed diagnosis. Testicular tumors presenting with gynecomastia may be nonpalpable, thus endocrine investigations are mandatory for all patients, even with unilateral gynecomastia.

Etiology of gynecomastia in testicular cancer is increased estrogen (i.e., by Leydig or Sertoli cell tumors or hCG-producing tumors). $95 \%$ of testicular tumors are germ cell tumors, and $40 \%$ of germ cell tumors are classic seminomas. These are most common in the fourth decade of life. Scattered syncytiotrophoblast elements are present in $7 \%$ of classic seminomas. These have been proposed to increase hCG level, which is a negative prognostic marker.
Patients with testicular tumors and gynecomastia were traditionally considered to have a worse prognosis and be at a later stage than men without this symptom, but this has not been confirmed by recent studies. In the case reported, gynecomastia led the patient to seek medical attention, which he would not have otherwise done.

This short report highlights the need for careful clinical examination and a high index of suspicion for all male patients presenting with a breast lump. Unlike breast cancer, which is extremely uncommon in young men, testicular cancer is the most common malignancy in this age group.

\title{
Nodular Papillomatosis in a 12-year-old Female
}

\author{
Julie M. Jorns, MD
}

Department of Pathology, University of Michigan, Ann Arbor, Michigan

A 12-year-old female presented with a 5-month history of self-detected right breast mass. Physical exam showed Tanner stage IV breast development with an $\sim 3 \mathrm{~cm}$ nodule in the lower outer quadrant of the right breast, $4 \mathrm{~cm}$ from the nipple. Ultrasound confirmed a solitary hypo-echoic irregular antiparallel mass (Fig. 1). The patient's past medical history was only significant for high body mass index (90\%) for age. Her first menses had occurred just prior to presentation.

Excisional biopsy revealed a $3.2 \times 3.1 \times 2.1 \mathrm{~cm}$ lobulated white-tan fibrous nodule. Histologic sections showed a relatively well-circumscribed nodule comprised of arborizing ducts with numerous intraductal papillomas with encircling sclerosis. The papillomas had varying degrees of usual ductal hyperplasia, ranging from mild to florid, but no significant atypia was present (Fig. 2).

Address correspondence and reprint requests to: Julie M. Jorns, MD, Department of Pathology, University of Michigan, 1500 East Medical Center Drive 2G332 UH, Ann Arbor, MI 48109, USA, or e-mail: jjorns@med.umich. edu

DOI: $10.1111 /$ tbj.12288

(C) 2014 Wiley Periodicals, Inc., 1075-122X/14

The Breast Journal, Volume 20 Number 4, 2014 426-427
Breast lesions in children and adolescents are relatively infrequent but the most commonly encountered include fibroadenoma, juvenile (virginal) hypertrophy and juvenile papillomatosis. Papillary duct hyperplasia is much rarer. In this case, the differential diagnosis included juvenile papillomatosis and papillary duct hyperplasia because of the presence of multiple intraductal papillomas. If the lesion had been associated with the nipple, the diagnosis of nipple adenoma may have also been considered as it frequently displays papillary proliferation.

A key feature of both juvenile papillomatosis and papillary duct hyperplasia is benign intraductal papillary

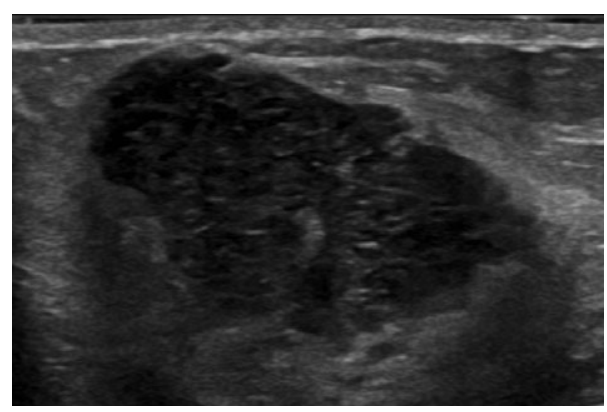

Figure 1. Ultrasound appearance of the irregular hypo-echoic mass. 

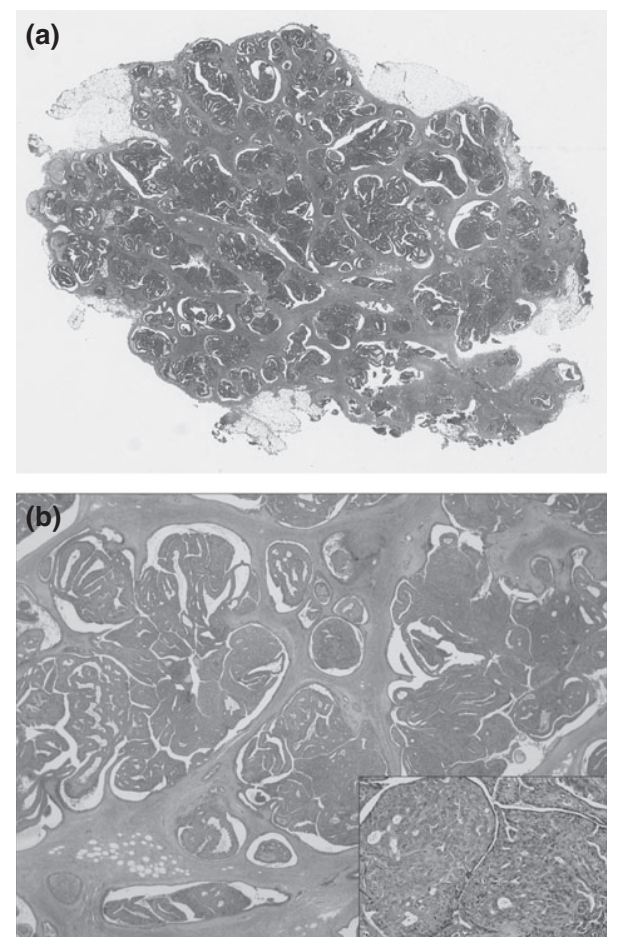

Figure 2. Nodular papillomatosis (H\&E, whole mount section) (a; $\mathrm{H} \& \mathrm{E}, 2 \times)$, (b) with inset showing prominent fibrovascular cores and benign usual ductal hyperplasia $(H \& E, 20 \times)$. proliferation. However, in juvenile papillomatosis the papillary proliferation is admixed with fibrocystic changes including cysts, apocrine metaplasia, usual ductal hyperplasia and other proliferative fibrocystic changes, which impart the classic "swiss cheese" appearance. These features were absent in this case, ruling out that diagnosis. Therefore, the diagnosis was most consistent with papillary duct hyperplasia, which lacks these additional features. Papillary duct hyperplasia involving multiple ducts, or papillomatosis, typically spreads along ducts linearly, often in the periphery of the breast. It does not usually form a discrete nodule as seen in this case, making this a very unusual presentation. The descriptive term "nodular papillomatosis" was used to convey this unusual benign diagnosis.

There are little data on the rare diagnosis of papillomatosis of the juvenile breast, but within the sparse literature there is evidence to support increased risk for future breast cancer. Thus, conservative resection with clinical follow-up to assess for recurrence as well as future breast disease is generally recommended. For this patient, it was felt that the mass had been adequately excised and further plans included clinical follow-up only.

\title{
Basal Cell Carcinoma of the Nipple
}

\author{
Ugur Ozerdem, MD, and Syed A. Hoda, MD
}

Pathology and Laboratory Medicine, Weill Cornell Medical College, New York, New York

A n 83-year-old woman presented with a palpable, otherwise asymptomatic, nodule on the left nipple. The patient had first noticed the nodule some 6-months previously, and had since observed its slow and gradual enlargement. Neither any pertinent medical nor family history was elicited.

On physical examination, there was a slightly raised nodule of the affected nipple. The skin overlying the

Address correspondence and reprint requests to: Ugur Ozerdem, MD, Pathology and Laboratory Medicine, Weill Cornell Medical College, $525 \mathrm{E}$ 68th Street, Starr 1036, New York, NY 10065, USA, or e-mail: uozerdem1@ gmail.com

DOI: $10.1111 /$ tbj.12290

(C) 2014 Wiley Periodicals, Inc., 1075-122X/14

The Breast Journal, Volume 20 Number 4, 2014 427-429 nodule was slightly darker in color. Both breasts and axillae were otherwise unremarkable. There were no mammographic abnormalities in either breast.

Excisional biopsy of the nipple revealed a tumor which was superficially invasive into the reticular dermis (Fig. 1a). The tumor was composed of large clusters of "basaloid" cells. The latter were cuboidal and hyperchromatic, with a high nuclear-cytoplasmic ratio. The tumor clusters showed cleft-like retraction from the adjacent stromal tissue (Fig. 1b). The tumor showed origin from the basal layer of the epidermis (Fig. 2a). The nipple epidermis was otherwise unremarkable. Anastomosing nests and cords of in the dermis characterized the tumor. There was peripheral 